\title{
Symptomatic and Asymptomatic Host Range of Fusarium virguliforme, the Causal Agent of Soybean Sudden Death Syndrome
}

\author{
T. M. Kolander, J. C. Bienapfl, J. E. Kurle, and D. K. Malvick, Department of Plant Pathology, University of Minnesota, St. Paul
} 55108

\begin{abstract}
Kolander, T. M., Bienapfl, J. C., Kurle, J. E., and Malvick, D. K. 2012. Symptomatic and asymptomatic host range of Fusarium virguliforme, the causal agent of soybean sudden death syndrome. Plant Dis. 96:1148-1153.

Sudden death syndrome, caused by Fusarium virguliforme, is an important disease of soybean in the United States. Fifteen species of crops, weeds, or prairie plants were evaluated for their potential as hosts of $F$. virguliforme. Root and foliar symptoms and plant biomass were assessed following greenhouse inoculation studies. Root colonization of $F$. virguliforme was determined with isolations and with polymerase chain reaction assays. Soybean, alfalfa, pinto and navy bean, white and red clover, pea, and Canadian milk vetch developed root necrosis. Soybean, alfalfa, and red clover also developed foliar symptoms following inoculation. Sugar beet and canola did not de-

velop symptoms but had significant reductions in biomass, suggesting that they are also hosts of $F$. virguliforme. Corn, wheat, ryegrass, pigweed, and lambsquarters did not develop symptoms. However, these species appeared to be asymptomatic hosts because quantities of pathogen DNA detected in inoculated roots were similar to quantities detected in inoculated soybean roots. These results suggest that the number and diversity of hosts for $F$. virguliforme are greater than previously reported. The likely broad host range limits the efficacy of crop rotation and indicates that crops other than soybean can be damaged by $F$. virguliforme and maintain or increase inoculum in soil.
\end{abstract}

Sudden death syndrome (SDS), caused by Fusarium virguliforme O'Donnell \& T. Aoki, is an important soilborne disease of soybean (Glycine max) that has been reported in at least 18 states in the United States $(2,4,15,23,26-28,40)$. In Minnesota, SDS was first reported in 2002 and has since been spreading and confirmed in 37 counties $(15,19$; D. K. Malvick, unpublished data). Symptoms of SDS include root necrosis, tan discoloration of vascular tissues in the lower stem, and interveinal leaf chlorosis and necrosis that progresses to premature defoliation (27). Environmental factors that favor the development of SDS include high soil moisture and cool soil temperatures during germination and vegetative growth $(7,27,30,32)$. In addition, the soybean cyst nematode (Heterodera glycines) may increase SDS severity $(25,27,29,32,39)$. Early planting, soil compaction, and irrigation can also increase the likelihood and severity of SDS $(7,27,29)$.

Management practices for reducing SDS include planting resistant cultivars, delayed planting, tillage, and possibly altering soil fertility $(7,12,27,30,32,35,37,39)$. Partial resistance to SDS is available in commercial cultivars and has been identified in soybean germplasm entries in the United States Department of Agriculture Soybean Germplasm collection; however, resistance is quantitative and most cultivars available in the United States are moderately resistant or susceptible to this disease $(11,19,22,24,25)$. Tillage may reduce SDS when soil is compacted and environmental conditions favor disease development $(27,35,38)$. Delayed planting can reduce the severity of SDS by allowing soil to dry and warm but may also limit yield potential (27). Altering soil nutrient quantities may reduce SDS but the effects have been inconsistent among field sites $(7,27,30,32)$.

Crop rotation, used to manage many soilborne pathogens, has had inconsistent effects on SDS severity and soil populations of $F$. virguliforme $(1,12,13,27,29,37,39)$. When compared with continuous soybean, a 3 -year sequence of wheat-corn-soybean reduced

Corresponding author: D. K. Malvick, E-mail: dmalvick@umn.edu

Accepted for publication 28 February 2012.

http://dx.doi.org/10.1094/PDIS-08-11-0685-RE

(C) 2012 The American Phytopathological Society
SDS severity in one study (37). Rotation of soybean with sorghum (Sorghum bicolor) or wheat reduced soil populations of F. virguliforme compared with continuous soybean, while rotations with fescue or corn resulted in soil populations similar to those found following continuous soybean $(29,39)$. Two-year crop sequences consisting of 1 year of soybean followed by 1 year of either corn, rice, or sorghum grain had little effect on reducing SDS severity $(12,13,27,39)$.

Although SDS is considered primarily to be a disease of soybean, $F$. virguliforme may infect other plants, and soil populations of this pathogen may be supported or maintained by crops planted in rotation with soybean $(10,21,29)$. The ineffectiveness of crop rotation for managing SDS also suggests that $F$. virguliforme may have a broader host range than reported in previous studies. Although the host range of $F$. virguliforme has not been studied extensively, several plant hosts that develop symptoms following inoculation have been reported. When inoculated with $F$. virguliforme without wounding, soybean, mung bean (Vigna radiate), and green bean (Phaseolus vulgaris) developed root rot, leaf chlorosis or necrosis and, in some cases, were stunted $(10,21)$. Following stem wounding with infested toothpicks, lima bean ( $P$. lunatus) and cowpea (V. unguiculata) developed brown stem lesions above and below the inoculation point and were considered to be compatible hosts (21). In addition to understanding which crops are at risk of infection and disease damage, it is critical to understand the range of asymptomatic plants capable of supporting the survival or reproduction of $F$. virguliforme. The objective of this study was to determine whether additional plant species commonly found or grown in the northern soybean-producing regions of the Midwestern United States are symptomatic or asymptomatic hosts of $F$. virguliforme.

\section{Materials and Methods}

Selection, inoculation, and maintenance of plant species. Fifteen species of crops, prairie plants, or weeds common to the northern regions of the Midwestern United States were evaluated as potential hosts of $F$. virguliforme (Table 1). Seed of all plant species was untreated, except that of canola, which was treated with thiamethoxam, difenoconazole, mefenoxam, and fludioxonil. The isolate of $F$. virguliforme (Wal-ss1) used for inoculation in these studies had been recovered from a soybean plant in Minne- 
sota expressing typical SDS symptoms. In previous studies, Wa1ss1 exhibited consistent pathogenicity on soybean and, thus, was chosen for determining the host range of $F$. virguliforme (19). The isolate was maintained in storage on carnation leaf agar and $2 \%$ water agar, and was transferred to half-strength potato dextrose agar (PDA), where it was grown for 1 week at $23^{\circ} \mathrm{C}$. The culture was increased by transferring to additional PDA plates, where it was grown for 2 weeks at $23^{\circ} \mathrm{C}$.

To produce inoculum, sorghum seed was placed in 250-ml flasks with water and allowed to soak approximately $24 \mathrm{~h}$. Then, the water was drained and the seed autoclaved twice and inoculated with $121-\mathrm{cm}^{2}$ plugs of the plate culture per flask $(19,22)$. The flasks were incubated for 2 to 3 weeks at $23^{\circ} \mathrm{C}$ under ambient fluorescent light with a 10 - to 11 -h photoperiod. Flasks were shaken daily to ensure uniform growth throughout the sorghum seed. Each plant species was inoculated in a greenhouse using a layer method $(19,22)$. A layer of $100 \mathrm{~cm}^{3}$ of either infested or noninfested sorghum seed for inoculated or noninoculated treatments, respectively, was placed on top of 750 to $1,500 \mathrm{~cm}^{3}$ of Sunshine LC-8 potting medium (Sun Gro Horticulture, Bellevue, WA), depending on planting depth of the specific plant species, in 13.7-cm-diameter square plastic pots (Belden Plastics, St. Paul, MN). The sorghum seed inoculum was covered with a $1-\mathrm{cm}$ buffer layer of $200 \mathrm{~cm}^{3}$ of potting medium and seed of potential hosts were placed on top of the buffer layer. Seed of soybean, corn, wheat, pinto bean, navy bean, ryegrass, pea, sugar beet, and canola were covered with potting medium followed by approximately $3 \mathrm{~mm}$ of sand to reduce damage from fungus gnats. Seed of alfalfa, pigweed, white clover, red clover, Canadian milk vetch, and lambsquarters were only covered with approximately $3 \mathrm{~mm}$ of sand to improve germination. Pots were arranged in a completely randomized design with four replications per treatment in each of two separate experiments. Osmocote 14-14$14\left(5 \mathrm{~cm}^{3} /\right.$ pot; The Scotts Co. LLC, Marysville, $\left.\mathrm{OH}\right)$ was added for all species except corn, where $10 \mathrm{~cm}^{3}$ was added. Plants were grown for approximately 5 weeks at $25^{\circ} \mathrm{C}$ (day) and $22^{\circ} \mathrm{C}$ (night) with a 14$\mathrm{h}$ photoperiod. Pots were watered regularly to maintain adequate moisture for plant growth and fertilized as needed with Peter's Professional 20-10-20 Peat-Lite Special (The Scotts Co.).

Pathogenicity tests. Disease symptoms and plant biomass were assessed approximately 5 weeks after planting. Foliar symptoms were evaluated using a 1-to-5 rating scale, where $1=$ no symptoms, $2=$ mottling and mosaic on 1 to $20 \%$ of the foliage, $3=$ interveinal chlorosis or necrosis on 21 to $50 \%$ of the foliage, $4=$ interveinal chlorosis or necrosis on 51 to $80 \%$ of the foliage, and $5=$ severe interveinal chlorosis or necrosis on 81 to $100 \%$ of the foliage (11). Roots were washed until soil was removed and disease severity was rated using a 1 -to- 5 scale, where $1=$ no symptoms, $2=$ brown lesions on 1 to $25 \%$ of the root system, $3=$ root rot on 25 to $49 \%$ of the root system, $4=$ root rot on 50 to $74 \%$ of the root system, and $5=$ root rot on $>75 \%$ of the root system (19). Fresh plant biomass was determined by weighing whole plants, including roots, stems, and leaves. The relative biomass of the inoculated plants was expressed as a percentage of the biomass of noninoculated plants of the same species.

Isolation and identification of $F$. virguliforme from inoculated plants. Taproots with the lateral roots removed from soybean, sugar beet, and pea, as well as taproots with lateral roots intact from the clovers, Canadian milk vetch, and alfalfa, were thoroughly rinsed a second time with tap water until all remaining debris trapped between the taproot and lateral roots was removed. Quartered corn roots and fully intact wheat and ryegrass roots were rinsed again until all accessible debris was dislodged, washed in a beaker with Tween 20 for $2 \mathrm{~min}$, and given a final rinse under running tap water for $5 \mathrm{~min}$. For isolations, four noninoculated and six inoculated plants per species in each experiment were arbitrarily chosen, and three segments of 0.5 to $1 \mathrm{~cm}$ in length were cut from each root. All segments for isolations were immersed in a $0.26 \%$ $\mathrm{NaOCl}$ solution for $1 \mathrm{~min}$, rinsed in sterile deionized water for 1 min, blotted dry, embedded in Modified Nash and Snyder's Medium (6), and incubated under ambient fluorescent light with a 10to 11 -h photoperiod at $25^{\circ} \mathrm{C}$ for 1 week. Cultures with morphological characteristics similar to $F$. virguliforme $(2,16)$ were transferred to acidified PDA $(\mathrm{pH}=5.0)$, then transferred to potato dextrose broth and grown at $23^{\circ} \mathrm{C}$ under ambient fluorescent light for 7 to 10 days (5). Genomic DNA was extracted from the mycelium and polymerase chain reaction (PCR) amplicons were generated using standard PCR (sPCR) with $F$. virguliforme-specific primers (Fsg1 and Fsg2) using either a Mastercycler 5331 (Eppendorf, Westbury, NY) or a PTC-100 Thermal Controller (MJ Research Inc., Waltham, MA) $(17,19)$. Isolates that yielded approximately 438-bp amplicons as detected with gel electrophoresis were identified as $F$. virguliforme. Replicated analysis of 1:10 dilutions of DNA from $F$. virguliforme mycelium ranging from $10 \mathrm{ng} / \mu \mathrm{l}$ to $100 \mathrm{fg} / \mu \mathrm{l}$ were tested to determine that the sensitivity of the sPCR assay was $F$.

Table 1. Plant species and varieties tested for susceptibility to infection by Fusarium virguliforme and their classification as symptomatic or asymptomatic hosts

\begin{tabular}{|c|c|c|c|}
\hline \multicolumn{2}{|l|}{ Plant species } & \multirow[b]{2}{*}{ Variety } & \multirow[b]{2}{*}{ Host $^{\mathrm{a}}$} \\
\hline Common name & Scientific name & & \\
\hline Soybean & Glycine $\max$ & AG2107 & $\mathrm{S}$ \\
\hline Corn & Zea mays & G-8745 & AS \\
\hline Alfalfa & Medicago sativa & Vernal & $\mathrm{S}$ \\
\hline Wheat & Triticum aestivum & Wheaton & AS \\
\hline Pinto bean & Phaseolus vulgaris & Maverick & $\mathrm{S}^{\mathrm{b}}$ \\
\hline Navy bean & P. vulgaris & Vista & $\mathrm{S}^{\mathrm{b}}$ \\
\hline Ryegrass & Lolium perenne & Gator III & AS \\
\hline Pea & Pisum sativum & Little Marvel & $S^{b}$ \\
\hline Pigweed & Amaranthus retroflexus & Unspecified & AS \\
\hline White clover & Trifolium repens & Alice & $S^{b}$ \\
\hline Red clover & T. pretense & Scarlet & $\mathrm{S}^{\mathrm{b}}$ \\
\hline Canadian milk vetch & Astragalus canadensis & Unspecified & $\mathrm{S}^{\mathrm{b}}$ \\
\hline Sugar beet & Beta vulgaris & SES VanderHave 46177 & $\mathrm{~S}^{\mathrm{c}}$ \\
\hline Lambsquarters & Chenopodium album & Unspecified & AS \\
\hline Canola & Brassica napus & DKL34-55 & $S^{c}$ \\
\hline
\end{tabular}

a Symptomatic (S) and asymptomatic (AS) hosts. Plant species were considered to be symptomatic hosts if average root or foliar disease severity ratings were $>2$ and were significantly greater than noninoculated plants of the same species. Plants were also considered symptomatic hosts if there was significant reduction in total plant biomass of inoculated plants compared with noninoculated plants. Plant species were considered to be asymptomatic hosts if disease severity was $<2$, there was reduction in biomass, $F$. virguliforme was detected in roots of the inoculated plants with isolations or with standard polymerase chain reaction, and quantities of $F$. virguliforme DNA detected in inoculated roots were within the range of quantities detected in inoculated soybean roots.

${ }^{\mathrm{b}}$ Inoculation caused root symptoms but not foliar symptoms.

${ }^{c}$ Inoculation resulted in significant reductions of total plant biomass in the absence of leaf chlorosis or necrosis and root rot. 
virguliforme DNA at $1 \mathrm{pg} / \mu \mathrm{l}$. For further confirmation of identity, the internal transcribed spacer (ITS) region of rDNA from five isolates was amplified with ITS1 and ITS4 primers, purified, and sequenced at the University of Minnesota BioMedical Genomics Center (St. Paul) (19). Isolates with $\geq 99 \%$ sequence similarity to $F$. virguliforme accessions deposited in the National Center for Biotechnology Information database (Bethesda, MD) by Aoki et al. (3) were confirmed as $F$. virguliforme.

Detection and quantification of $F$. virguliforme in plant tissue using PCR. Root tissue collected from each pot was combined and ground for extraction, with the exception of fine root systems, where the roots from multiple pots were aggregated (e.g., Canadian milk vetch, alfalfa, clovers, and other root systems that yielded limited tissue). DNA was extracted from $100 \mathrm{mg}$ of ground root tissue, with the exception of corn, where $66 \mathrm{mg}$ was used $(19,20)$. Extractions were completed from each of two inoculated and noninoculated pots for each plant species in each of two experiments. DNA of $F$. virguliforme was detected in roots with SPCR reactions as described above, based on the presence or absence of approximately 438-bp amplicons. The amount of $F$. virguliforme DNA in roots was determined by quantitative real-time (q)PCR using an Applied Biosystems 7500 Real-Time PCR System (Foster City, $\mathrm{CA})$. The reactions contained $5 \mu \mathrm{l}$ of $1 \times$ DNA from roots, $12.5 \mu \mathrm{l}$ of Taqman Universal PCR Master Mix (Applied Biosystems), 450 $\mathrm{nM}$ each of Fsg-q-1 forward and reverse primers, $200 \mathrm{nM}$ probe, and $2.75 \mu \mathrm{l}$ of molecular-grade water (9). Duplicate qPCR reactions were completed for each sample. The quantities of $F$. virguliforme DNA in roots were calculated using a standard curve, generated from 10 -fold serial dilutions ranging from $20 \mathrm{ng} / \mu \mathrm{l}$ to $2 \mathrm{fg} / \mu \mathrm{l}$, and normalized to $100 \mathrm{mg}$ of tissue.

Symptomatic and asymptomatic hosts. Plant species were considered to be symptomatic hosts if average root or foliar disease severity ratings were $>2$ and significantly greater than noninoculated plants of the same species in the same experiment. A threshold of 2 was chosen because factors other than infection with $F$. virguliforme sometimes caused low levels of root or leaf discoloration. In addition, plants were considered symptomatic hosts in the absence of leaf or root symptoms if there was a significant reduction in plant biomass of inoculated plants compared with noninoculated plants of the same species. Plant species were considered to be asymptomatic hosts if (i) disease severity was $<2$, (ii) there was no significant reduction in plant biomass, (iii) $F$. virguliforme was detected in roots of the inoculated plants with isolations or with SPCR, and (iv) quantities of $F$. virguliforme DNA detected in inoculated roots were within the range of quantities detected in inoculated soybean roots.

Table 2. Average foliar disease severity ratings (1-to- 5 scale, where $1=$ no symptoms and $5=$ severe interveinal chlorosis or necrosis on 81 to $100 \%$ of the foliage) of plant species inoculated with Fusarium virguliforme

\begin{tabular}{lcc}
\hline \multirow{2}{*}{ Plant species } & \multicolumn{2}{c}{ Average foliar disease severity ratings $^{\mathbf{a}}$} \\
\cline { 2 - 3 } Experiment 1 & Experiment 2 $^{*}$ \\
\hline Soybean & $3.6^{*}$ & $3.9^{*}$ \\
Corn & 1.0 & 1.0 \\
Alfalfa & 1.1 & $4.4^{*}$ \\
Wheat & 1.0 & 1.0 \\
Pinto bean & 1.0 & 1.4 \\
Navy bean & 1.0 & $1.6^{*}$ \\
Ryegrass & 1.0 & 1.0 \\
Pea & 1.0 & 1.2 \\
Pigweed & 1.1 & 1.0 \\
White clover & 1.2 & 1.5 \\
Red clover & 1.4 & $2.5^{*}$ \\
Canadian milk vetch & 2.0 & 1.0 \\
Sugar beet & 1.0 & 1.0 \\
Lambsquarters & 1.3 & 1.0 \\
Canola & 1.0 & 1.0 \\
\hline
\end{tabular}

${ }^{a}$ Asterisk $(*)$ indicates that the average foliar disease severity ratings for inoculated plants was significantly $(\alpha=0.05)$ greater than that of noninoculated plants in the same experiment.
Statistical analysis. Treatments were arranged differently in repeated greenhouse pathogenicity experiments; thus, the experiments were separated for subsequent analyses of nontransformed data. A $t$ test was performed on root and foliar disease ratings using the Fit $\mathrm{Y}$ by $\mathrm{X}$ function in JMP (JMP version 9.0.2; SAS Institute Inc., Cary, NC) to determine whether either root or foliar disease ratings differed significantly between inoculated and noninoculated plants of the same species $(P \leq 0.05)$. A PROC TTEST (SAS version 9.2; SAS Institute Inc.) was used to test for significant differences $(P \leq 0.10)$ between biomass of inoculated and noninoculated treatments within each species.

\section{Results}

Pathogenicity test results. Inoculation with $F$. virguliforme resulted in significant root rot, leaf necrosis and chlorosis, and reduced plant biomass for multiple plant species when compared with noninoculated plants of the same species. Inoculated soybean plants, included as positive host controls, developed foliar disease with average ratings $>3$ and root rot with average ratings $>4$ in all experiments (Tables 2 and 3). Inoculated alfalfa and red clover developed significant foliar disease, with average severity ratings $>2$ and symptoms consisting of stem and leaf necrosis, stunting, and premature foliar death in one of two experiments (Table 2). In addition, alfalfa and red clover consistently developed severe root rot in both experiments (Table 3). Inoculated pinto bean, navy bean, pea, white clover, and Canadian milk vetch also developed significant root rot ratings $>2$ in one or more experiments (Table 3). The other seven plant species tested did not develop significant root or foliar symptoms $>2$. Biomass of inoculated soybean, alfalfa, pinto bean, navy bean, white clover, red clover, Canadian milk vetch, sugar beet, and canola was significantly reduced in comparison with noninoculated controls in at least one experiment (Fig. 1).

$F$. virguliforme was isolated at least once from inoculated roots from most species, and the identity of the isolates was confirmed based on culture morphology, specific sPCR amplicons, and ITS sequences. This fungus was not isolated from noninoculated control roots. Using sPCR, $F$. virguliforme DNA was detected in 83 to $100 \%$ of roots from inoculated soybean, corn, alfalfa, wheat, pinto bean, navy bean, ryegrass, pea, pigweed, white clover, red clover, Canadian milk vetch, sugar beet, canola and lambsquarters (Fig. 2 ). DNA from $F$. virguliforme was not detected with SPCR in most noninoculated control samples, except for a few Canadian milk vetch and red clover.

Quantification of $F$. virguliforme in plant tissue using qPCR. A standard curve was developed and used to determine the amount of $F$. virguliforme DNA present in the roots of all plant species

Table 3. Average root disease severity rating (1-to-5 scale, where $1=$ no symptoms and $5=$ root rot on $>75 \%$ of the root system) of plant species inoculated with Fusarium virguliforme

\begin{tabular}{lcc}
\hline & \multicolumn{2}{c}{ Average root disease severity ratings $^{\mathbf{a}}$} \\
\cline { 2 - 3 } Plant species & Experiment 1 & Experiment 2 $^{*}$ \\
\hline Soybean & $4.3^{*}$ & $4.5^{*}$ \\
Corn & 2.0 & 1.5 \\
Alfalfa & $3.1^{*}$ & $5.0^{*}$ \\
Wheat & 1.8 & 1.8 \\
Pinto bean & 3.5 & $4.4^{*}$ \\
Navy bean & $2.6^{*}$ & $4.1^{*}$ \\
Ryegrass & 1.0 & 1.3 \\
Pea & $1.9^{*}$ & $3.9^{*}$ \\
Pigweed & 1.1 & 1.0 \\
White clover & $2.9^{*}$ & $3.7^{*}$ \\
Red clover & $4.4^{*}$ & $3.8^{*}$ \\
Canadian milk vetch & $4.7^{*}$ & 1.1 \\
Sugar beet & 1.2 & 1.9 \\
Lambsquarters & 1.0 & 1.0 \\
Canola & 1.5 & 1.1 \\
\hline
\end{tabular}

${ }^{a}$ Asterisk $(*)$ indicates that the average root disease ratings for inoculated plants was significantly greater $(\alpha=0.05)$ than that of noninoculated plants in the same experiment. 
tested. The standard curve was developed using linear regression (Microsoft Excel 2007, Redmond, WA) to obtain the formula $y=$ $-3.4737 x+41.116\left(R^{2}=0.99\right)$, where $x=$ the log DNA concentration, and $y=$ cycle threshold $(\mathrm{Ct})$ value. This formula was used to calculate the quantity of $F$. virguliforme DNA present in the tissue. The reaction efficiency was approximately $95 \%$, sensitivity was $F$. virguliforme DNA at $0.2 \mathrm{pg} / \mu \mathrm{l}$, and the limit of detection was $\mathrm{Ct}=32.6(9,34)$. The quantity of $F$. virguliforme DNA detected varied in the roots of all plant species tested (Fig. 3). All inoculated species that developed any combination of root symptoms, leaf symptoms, or a decrease in total plant biomass, including alfalfa, pinto bean, navy bean, pea, white clover, red clover, Canadian milk vetch, sugar beet, and canola, contained quantities of $F$. virguliforme DNA in their roots that were within the range detected in inoculated soybean root tissue (Fig. 3A). In addition, all species not expressing any SDS symptoms, including corn, wheat, ryegrass, pigweed, and lambsquarters, contained quantities of $F$. virguliforme DNA in their roots that overlapped with that detected in soybean roots (Fig. 3B).

Symptomatic and asymptomatic host range of $F$. virguliforme. Based on the results and criteria described above, 10 of the 15 plant species tested were symptomatic hosts and five species were asymptomatic hosts of $F$. virguliforme (Table 1 ). Only alfalfa, red clover, and soybean developed stem or leaf necrosis and root rot in at least one experiment. Pinto bean, navy bean, pea, white clover, and Canadian milk vetch developed only root rot in one or more experiments. Sugar beet and canola were also classified as symptomatic hosts in the absence of observable root rot or leaf chlorosis or necrosis because a significant loss of biomass occurred in at least one experiment when plants were inoculated. Corn, wheat, ryegrass, pigweed, and lambsquarters were considered to be asymptomatic hosts because (i) they did not develop leaf chlorosis or necrosis, root rot, or losses in total plant biomass; (ii) F. virguli- forme was detected in roots by isolation or with SPCR; and (iii) quantities of $F$. virguliforme DNA detected in roots with qPCR were similar to that detected in soybean roots.

\section{Discussion}

SDS is an important soybean disease that is difficult to manage. Crop rotations typically have little or no effect on SDS or on soil populations of $F$. virguliforme $(12,13,27,29,37,39)$. The published host range for $F$. virguliforme is limited to five plant species $(10,21)$ but a more complete understanding of its host range may help to explain both why crop rotation has limited efficacy for managing SDS and whether other plant species can be infected and damaged by $F$. virguliforme.

Most of the symptomatic hosts that were tested in this study developed root rot or root discoloration but few developed chlorosis or necrosis of leaves or stems. Thus, it is important to note that the symptoms on hosts other than soybean often differed from those caused by $F$. virguliforme on soybean. Both root and foliar symptoms were observed only on inoculated soybean, alfalfa, and red clover. Foliar symptoms were produced consistently on soybean but were observed inconsistently on alfalfa and red clover. Root symptoms were observed consistently on alfalfa, navy bean, white clover, and red clover, and inconsistently on pinto bean, pea, and Canadian milk vetch. The inconsistency may suggest that these species are not as susceptible to infection as the other hosts under the experimental conditions used in this research, or it may reflect partial or full escape from the inoculation treatment. Inconsistent symptom expression has also been reported in tests for varietal resistance to SDS in soybean $(21,22)$. The development of foliar or root symptoms suggests that these important crops and forages may be adversely affected by this pathogen, albeit primarily due to root rot, which differs from the combined root rot and foliar chlorosis and necrosis typically seen in soybean.

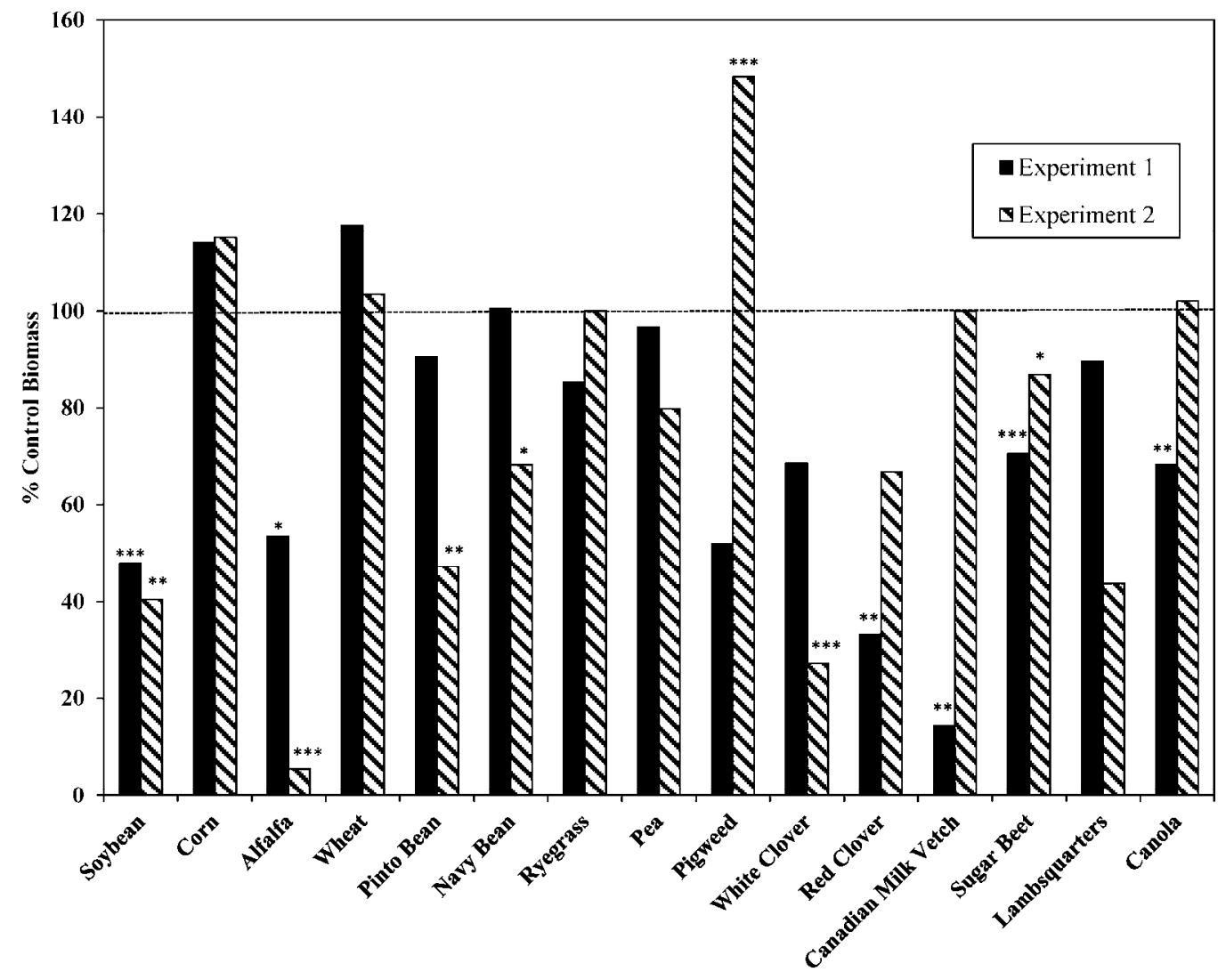

Plant Species - Common Name

Fig. 1. Effect of inoculation on biomass of 15 plant species evaluated for susceptibility to infection by Fusarium virguliforme. Biomass of inoculated plants is expressed as a percentage of biomass of noninoculated plants. Values represent the average of four replications in two experiments. Differences between inoculated and noninoculated plants of each species were analyzed with a $t$ test, where ${ }^{*},{ }^{* *}$, and ${ }^{* * *}$ indicate $P \leq 0.10,0.05$, and 0.01 , respectively. 
Sugar beet and canola are also classified as symptomatic hosts due to the significant reduction in total plant biomass following inoculation. Neither of these plant species developed root rot or leaf chlorosis or necrosis. Sugar beet was stunted in both experiments while canola was significantly stunted in one of two experiments. These results suggest that sugar beet and canola could also suffer damage and yield loss caused by $F$. virguliforme.

A plant species was considered to be an asymptomatic host if, when inoculated, it did not develop foliar symptoms or significant reductions in biomass, $F$. virguliforme was detected in roots by isolation or by sPCR assays, and roots contained quantities of $F$. virguliforme DNA similar to the amounts detected in inoculated soybean roots. Corn, wheat, ryegrass, pigweed, and lambsquarters were considered to be asymptomatic hosts. Foliar, root, or stunting symptoms did not develop on the leaves or roots of these species following inoculation with $F$. virguliforme. However, $F$. virguliforme was detected in roots of all five species by isolation or by sPCR assays, and they contained quantities of $F$. virguliforme DNA in roots similar to the amounts detected in soybean roots with qPCR. The results obtained with corn are especially important

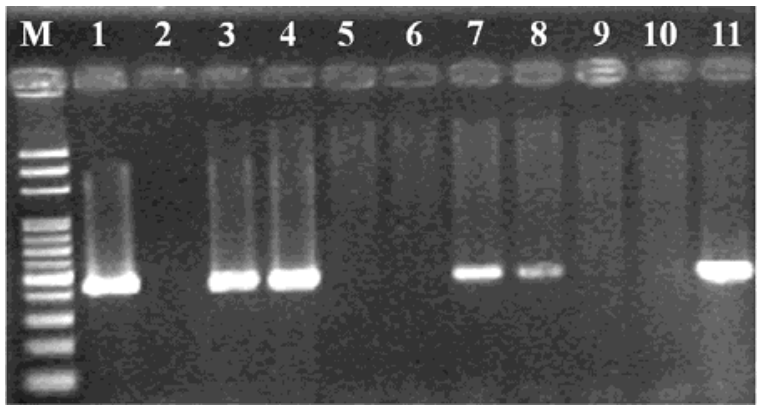

Fig. 2. Detection of Fusarium virguliforme DNA from inoculated roots with standard polymerase chain reaction (PCR) using species-specific primers. The amplicon is approximately 438 bp. Lane M, 100-bp ladder; lane 1, positive PCR control containing F. virguliforme DNA; lane 2, non-template PCR control; lanes 3 and 4, inoculated canola; lanes 5 and 6 , noninoculated sugar beet; lanes 7 and 8 , inoculated sugar beet; lanes 9 and 10, noninoculated alfalfa; lane 11, inoculated alfalfa. because this crop is rotated with soybean in most production areas and rotation of corn with soybean crops does not suppress SDS $(27,39)$. These results suggest that $F$. virguliforme may infect or colonize corn asymptomatically and that corn may support growth and reproduction of this pathogen, resulting in maintenance or increase in inoculum density. Similarly, wheat harbored $F$. virguliforme without visible symptoms.

F. virguliforme was reisolated from inoculated soybean, corn, alfalfa, wheat, pinto bean, navy bean, ryegrass, pigweed, white clover, red clover, Canadian milk vetch, lambsquarters, and canola in this study. This confirms that $F$. virguliforme can be cultured and remains viable in both symptomatic and asymptomatic host tissue. $F$. virguliforme was sometimes difficult to isolate because other, faster-growing fungi from infected roots often overgrew it. Difficulty in isolating $F$. virguliforme has been reported previously due to its relatively slow growth, the availability of only a semiselective medium, and its morphological similarity with other Fusarium spp. $(6,16,28)$.

PCR is an effective tool for detection and quantification of fungi in plant tissue $(9,31,36)$. sPCR was used to confirm the presence of $F$. virguliforme in inoculated root systems and to confirm its absence in noninoculated plant roots. Data obtained using SPCR augmented that obtained by direct isolation of $F$. virguliforme on media. Detection of $F$. virguliforme with SPCR in noninoculated roots confirmed its presence but did not distinguish between infection and potential surface contamination, as likely happened with a few samples of Canadian milk vetch and red clover that had small root systems and minimal capability of diluting contamination. qPCR was the more informative technique. By quantifying the amount of $F$. virguliforme DNA present in washed plant roots, it provided a measure of reproduction for $F$. virguliforme in inoculated roots with and without root disease symptoms. The large quantities of pathogen DNA detected with qPCR in roots of inoculated host plants compared with noninoculated controls distinguished reproduction and growth from surface contamination as a source of pathogen DNA. When the quantity of pathogen DNA detected in a plant species overlapped with the range of quantities detected in inoculated soybean, it suggested that the species supported pathogen colonization levels similar to those in soybean. Thus, qPCR
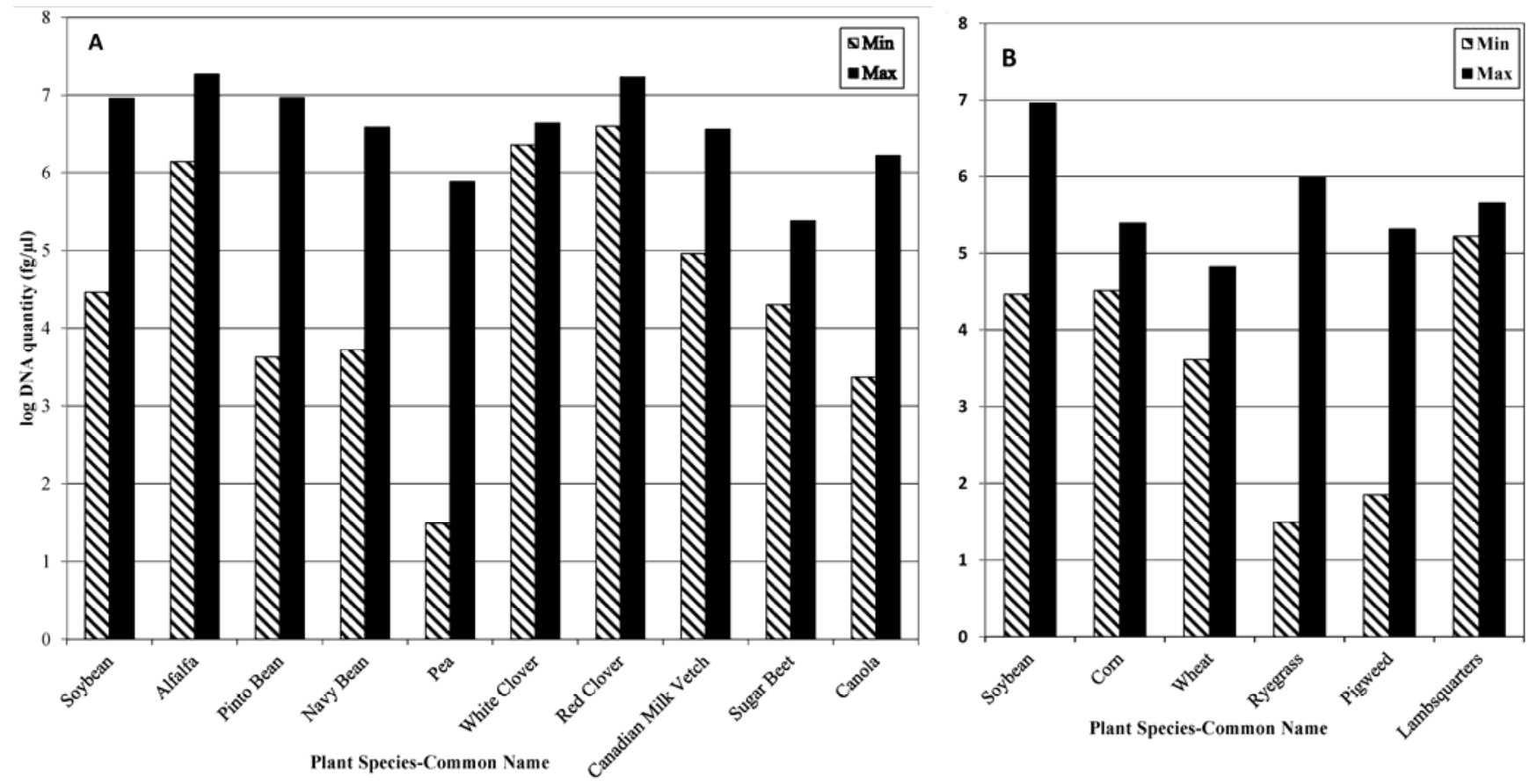

Fig. 3. Minimum and maximum quantity of Fusarium virguliforme DNA detected in inoculated roots of $\mathbf{A}$, symptomatic and $\mathbf{B}$, asymptomatic plant species using an $F$. virguliforme-specific, quantitative real-time polymerase chain reaction (qPCR) assay. Soybean is shown as a symptomatic check in B for comparison. Each black and diagonal-striped vertical bar represents the maximum and minimum amount, respectively, of $F$. virguliforme DNA detected in two separate samples in each of two experiments. The limit of detection for $F$. virguliforme DNA for the qPCR assays was $\log 2.3 \mathrm{fg} / \mu \mathrm{l}$. 
provided strong evidence that $F$. virguliforme reproduced in and colonized roots of the asymptomatic hosts, including corn.

This study demonstrates that multiple plant species can serve as hosts of $F$. virguliforme. However, additional studies with lower amounts of inoculum and conducted under natural field conditions could help to clarify the susceptibility of these species. The inoculum dose and substrate used in this study potentially could have allowed $F$. virguliforme to colonize and infect species that are nonhosts in field or low-inoculum conditions. The rigorous washing procedures and isolation techniques provide evidence that the pathogen penetrated the root tissue of plants classified as asymptomatic hosts. This study also provides a novel perspective into a possible means of survival for $F$. virguliforme via a nondestructive infection of roots in the absence of soybean.

The results of this study indicate that the host range of $F$. virguliforme should be expanded to include the 10 symptomatic and 5 asymptomatic hosts reported in this study. This expanded host range for $F$. virguliforme helps to explain why crop rotation has not been effective for managing SDS in soybean and also indicates which plant species may increase or maintain soil populations of this pathogen when grown in rotation with soybean. The symptomatic hosts may also be at risk of disease damage and yield loss from this pathogen. Many weeds and prairie plants that are found in agricultural fields should be managed because they may harbor $F$. virguliforme and increase inoculum in soil. Similar results have been reported previously with other Fusarium spp., indicating that weeds can act as hosts that harbor fungal populations $(8,14,16,18,33)$. The results also suggest that management strategies other than crop rotation should be a priority for reducing yield losses due to $F$. virguliforme in soybean and other crops. Additional research is needed to understand how $F$. virguliforme colonizes and survives on asymptomatic hosts, the impact of crop rotation, and whether the symptoms, colonization, and biomass reductions detected in greenhouse experiments also occur under field conditions.

\section{Acknowledgments}

We thank the Minnesota Rapid Agricultural Response Fund, the Minnesota Soybean Research and Promotion Council, and the University of Minnesota Agricultural Experiment Station for funding to support this project; and J. Eichmiller, C. Floyd, D. Foster, M. Holland, L. Kinkel, C. Kistler, P. Manlick, J. Merriman, M. Murtaugh, L. Otto-Hanson, M. Pilsner, N. Powers, M. Sadowsky, A. Testen, and those who provided seed for their academic, technical, and research support.

\section{Literature Cited}

1. Agrios, G. N. A. 2005. Plant Pathology, 5th ed. Elsevier Academic Press, Burlington, MA.

2. Aoki, T., O'Donnell, K., Homma, Y., and Lattanzi, A. R. 2003. Suddendeath syndrome of soybean is caused by two morphologically and phylogenetically distinct species within the Fusarium solani species complex-F. virguliforme in North America and F. tucumaniae in South America. Mycologia 95:660-684.

3. Aoki, T., O’Donnell, K., and Scandiani, M. M. 2005. Sudden death syndrome of soybean in South America is caused by four species of Fusarium: Fusarium brasiliense sp. nov., F. cuneirostrum sp. nov., F. tucumaniae, and F. virguliforme. Mycoscience 46:162-183.

4. Bernstein, E. R., Atallah, Z. K., Koval, N. C., Hudelson, B. D., and Grau, C. R. 2007. First report of sudden death syndrome of soybean in Wisconsin. Plant Dis. 91:1201-1201.

5. Bienapfl, J. C., Ocamb, C. M., Klein, R., and Nelson, M. 2005. Fusarium cone tip blight of Humulus lupulus. Acta Hortic. 668:123-128.

6. Cho, J. H., Rupe, J. C., Cummings, M. S., and Gbur, E. E. 2001. Isolation and identification of Fusarium solani $\mathrm{f}$. sp. glycines from soil on modified Nash and Snyder's medium. Plant Dis.85:256-260.

7. Chong, S. K., Hildebrand, K. K., Luo, Y., Myers, O., Indorante, S. J., Kazakevicius, A., and Russin, J. 2005. Mapping soybean sudden death syndrome as related to yield and soil/site properties. Soil Tillage Res. 84:101-107.

8. Correll, J. C., Puhalla, J. E., and Schneider, R. W. 1986. Identification of Fusarium oxysporum f. sp. apii on the basis of colony size, virulence, and vegetative compatibility. Phytopathology 76:396-400.

9. Gao, X., Jackson, T. A., Lambert, K. N., Li, S., and Hartman, G. L., and Niblack, T. L. 2004. Detection and quantification of Fusarium solani f. sp. glycines in soybean roots with real-time quantitative polymerase chain reaction. Plant Dis. 88:1372-1380.

10. Gray, L. E., Achenbach, L. A., Duff, R. J., and Lightfoot, D. 1999. Pathogenicity of Fusarium solani f. sp. glycines isolates on soybean and green bean plants. J. Phytopathol. 147:281-284.
11. Hartman, G. L., Huang, Y. H., Nelson, R. L., and Noel, G. R. 1997. Germplasm evaluation of Glycine max for resistance to Fusarium solani, the causal organism of sudden death syndrome. Plant Dis. 81:515-518.

12. Hirrel, M. C. 1986. Disease severity and yield loss comparisons of soybean maturity groups affected in sudden death syndrome. Proceedings of Southern Soybean Disease Workers 13th Annual Meeting 15:61.

13. Hirrel, M. C. 1986. Sudden death syndrome of soybean: new insights into its development. Pages 95-104 in: Proc. 16th Soybean Seed Res. Conf.

14. Kedera, C. J., Leslie, J. F., and Claflin, L. E. 1994. Genetic diversity of Fusarium section Liseola (Gibberella fujikuroi) in individual maize stalks. Phytopathology 84:603-607.

15. Kurle, J. E., Gould, S. L., Lewandowski, S. M., Li, S., and Yang. X. B. 2003. First report of sudden death syndrome (Fusarium solani f. sp. glycines) of soybean in Minnesota. Plant Dis. 87:449.

16. Leslie, J. F., and Summerell, B. A. 2006. The Fusarium Laboratory Manual, 1 st ed. Blackwell Pub, Ames, IA.

17. Li, S., and Hartman, G. L. 2003. Molecular detection of Fusarium solani f. sp. glycines in soybean roots and soil. Plant Pathol. 52:74-83.

18. MacDonald, J. D., and Leach, L. D. 1976. Evidence for an expanded host range of Fusarium oxysporum f. sp. betae. Phytopathology 66:822-827.

19. Malvick, D. K., and Bussey, K. E. 2008. Comparative analysis and characterization of the soybean sudden death syndrome pathogen Fusarium virguliforme in the northern United States. Can. J. Plant Pathol. 30:467-476.

20. Malvick, D. K., and Grunden, E. 2005. Isolation of fungal DNA from plant tissues and removal of DNA amplification inhibitors. Mol. Ecol. Notes 5:958-960.

21. Melgar, J., and Roy, K. W. 1994. Soybean sudden-death syndrome - cultivar reactions to inoculation in a controlled environment and host-range and virulence of causal agent. Plant Dis. 78:265-268.

22. Mueller, D. S., Nelson, R. L., Hartman, G. L., and Pedersen, W. L. 2003. Response of commercially developed soybean cultivars and the ancestral soybean lines to Fusarium solani f. sp. glycines. Plant Dis. 87:827-831.

23. Mulrooney, R. P., Gregory, N. F., Walker, S. D., and Webster, A.-M. 2002. First report of sudden death syndrome of soybean in Delaware and eastern shore of Maryland. Plant Dis. 86:696.

24. Njiti, V. N., Doubler, T. W., Suttner, R. J., Gray, L. E., Gibson, P. T., and Lightfoot, D. A. 1998. Resistance to soybean sudden death syndrome and root colonization by Fusarium solani f. sp. glycines in near-isogenic lines. Crop Sci. 38:472-477.

25. Njiti, V. N., Shenaut, M. A., Suttner, R. J., Schmidt, M. E., and Gibson, P. T. 1996. Soybean response to sudden death syndrome: inheritance influenced by cyst nematode resistance in Pyramid $\times$ Douglas progenies. Crop Sci. 36:1165-1170.

26. Pennypacker, B. W. 1983. First report of sudden death syndrome caused by Fusarium solani f. sp. glycines on soybean in Pennsylvania. Plant Dis. 83:879.

27. Roy, K. W., Rupe, J. C., Hershman, D. E., and Abney, T. S. 1997. Sudden death syndrome of soybean. Plant Dis. 81:1100-1111.

28. Rupe, J. C. 1989. Frequency and pathogenicity of Fusarium solani recovered from soybeans with sudden death syndrome. Plant Dis. 73:581-584.

29. Rupe, J. C., Robbins, R. T., and Gbur, E. E. 1997. Effect of crop rotation on soil population densities of Fusarium solani and Heterodera glycines and on the development of sudden death syndrome of soybean. Crop Prot. 16:575-580.

30. Rupe, J. C., Sabbe, W. E., Robbins, R. T., and Gbur, E. E. 1993. Soil and plant factors associated with sudden-death syndrome of soybean. J. Prod. Agric. 6:218-221.

31. Schaad, N. W., and Frederick, R.D. 2002. Real-time PCR and its application for rapid plant disease diagnosis. Can. J. Plant Pathol. 24:250-258.

32. Scherm, H., Yang, X. B., and Lundeen, P. 1998. Soil variables associated with sudden death syndrome in soybean fields in Iowa. Plant Dis. 82:11521157.

33. Smith, S. N., and Snyder, W. C. 1975. Persistence of Fusarium oxysporum f. sp. vasinfectum in fields in absence of cotton. Phytopathology 65:190-196.

34. Stratagene Products Division. 2007. Introduction to Quantitative PCR: Methods and Applications Guide. Agilent Technologies, Inc., Santa Clara, CA.

35. Vick, C. M., Bond, J. P., Chong, S. K., and Russin, J. S. 2006. Response of soybean sudden death syndrome to tillage and cultivar. Can. J. Plant Pathol. 28:77-83.

36. Vincelli, P., and Tisserat, N. 2008. Nucleic acid-based pathogen detection in applied plant pathology. Plant Dis. 92:660-669.

37. Von Qualen, R. H., Abney, T. S., Huber, D. M., and Schreiber, M. M. 1989. Effects of rotation, tillage, and fumigation on premature dying of soybeans. Plant Dis. 73:740-744.

38. Wrather, J. A., Kendig, S. R., Anand, S. C., Niblack, T. L., and Smith, G. S. 1995. Effects of tillage, cultivar, and planting date on percentage of soybean leaves with symptoms of sudden-death syndrome. Plant Dis. 79:560-562.

39. Xing, L. J., and Westphal, A. 2009. Effects of crop rotation of soybean with corn on severity of sudden death syndrome and population densities of Heterodera glycines in naturally infested soil. Field Crops Res. 112:107-117.

40. Ziems, A. D., Giesler, L. J., and Yuen, G. Y. 2006. First report of sudden death syndrome of soybean caused by Fusarium solani f. sp. glycines in Nebraska. Plant Dis. 90:109-109. 